\title{
Popliteal Vein
}

National Cancer Institute

\section{Source}

National Cancer Institute. Popliteal Vein. NCI Thesaurus. Code C33339.

A vein originating from the anterior and posterior tibial veins that ascends the popliteal space to drain blood from the calf, knee joint, and thigh. The popliteal vein ultimately becomes the femoral vein. 